KAMINISHI, A.P.S. et al. Comportamento da artéria aorta em Trachemys dorbigni (Duméril \& Bibron, 1835) (Testudines, Emydidae). PUBVET, Londrina, V. 5, N. 10, Ed. 157, Art. $1061,2011$.

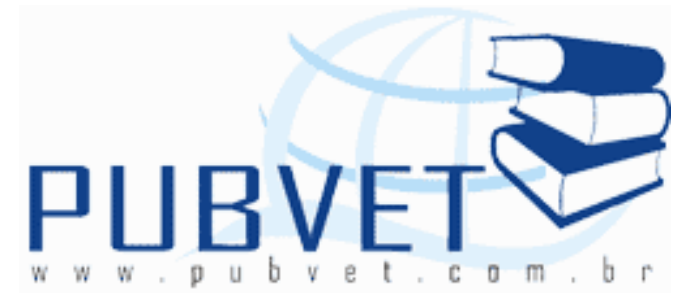

PUBVET, Publicações em Medicina Veterinária e Zootecnia.

\title{
Comportamento da artéria aorta em Trachemys dorbigni (Duméril \& Bibron, 1835) (Testudines, Emydidae)
}

Árthur Paulino Sano Kaminishi ${ }^{2}$, André Luiz Quagliatto Santos ${ }^{1}$, Carlos Gomes Ferreira ${ }^{1}$, Líria Queiroz Luz Hirano ${ }^{2}$, Juliana Macedo Magnino Silva ${ }^{2}$

1. Docente da Faculdade de Medicina Veterinária, Universidade Federal de Uberlândia (FAMEV/UFU). 2. Mestrando em Ciências Veterinárias, FAMEV/UFU.

\section{Resumo}

O objetivo desse trabalho foi descrever o comportamento da artéria aorta, focalizando a origem, trajeto e o destino de suas ramificações. Utilizaram-se dez exemplares de Trachemys dorbigni, de ambos os sexos. A artéria aorta dos exemplares foi canulada para a introdução de solução fisiológica a fim de desobstruir o sistema vascular, em seguida, aplicou-se solução de Neoprene Látex "450" corada. Fixou-se o material em solução de Formol $10 \%$ por um período de 96 horas, após este, desprendeu-se o plastrão de suas inserções, evidenciando as vísceras e posteriormente individualizou-se a artéria aorta. Os arcos aórticos direito e esquerdo unem-se em um ponto após sua origem no coração. O arco aórtico esquerdo emite a artéria gástrica, que surge cranialmente ao ponto de união dos arcos aórticos, tal como a artéria celíaca, 
KAMINISHI, A.P.S. et al. Comportamento da artéria aorta em Trachemys dorbigni (Duméril \& Bibron, 1835) (Testudines, Emydidae). PUBVET, Londrina, V. 5, N. 10, Ed. 157, Art. $1061,2011$.

que fornece as artérias pancreaticoduodenais cranial e caudal e a artéria mesentéricas cranial. O tronco formado pela união dos dois arcos aórticos, denominado artéria aorta origina as artérias renais, epigástricas, ilíacas comuns e a artéria caudal. $\mathrm{O}$ arco aórtico direito não emite nenhum ramo antes da sua união com o arco aórtico esquerdo, portanto, os principais ramos viscerais surgem do arco aórtico esquerdo ou diretamente da artéria aorta.

Palavras-chave: Arco aórtico, morfologia, tigre d'água brasileiro

\title{
Aorta artery behaviour of the Trachemys dorbigni (Duméril \& Bibron, 1835) (Testudinata- Emydidae)
}

\begin{abstract}
The aim of this study was to describe the behaviour of the aorta artery, to focus its origin, trajectory, and destiny of its ramifications. There were used ten females and males turtles. We canalized the common carotid arteries through which we introduced a physiological solution to remove obstruction from the vascular system, than a colored solution of Neoprene Latex "450" was injected. We fixated the material in Formaldehyde $10 \%$ for a period of 96 hours. After this, we removed the skull from its insertions, showing the viscera and individualizing the aorta artery. We observed that the right and left aortic arch unite in a point right after its origin in the heart. The left aortic arc send the gastric artery, celiac artery and the cranial mesenteric artery which arise cranial to the point of union of the aortic arcs. It still originates a celiac artery that provides the cranial pancreaticduodenal artery. Right after, the trunk formed by the union of the two aortic arch is denominated the aorta artery and from it originates the renal, epigastrics, external iliac, internal iliac and the caudal arteries, that is a thin segment of
\end{abstract}


KAMINISHI, A.P.S. et al. Comportamento da artéria aorta em Trachemys dorbigni (Duméril \& Bibron, 1835) (Testudines, Emydidae). PUBVET, Londrina, V. 5, N. 10, Ed. 157, Art. $1061,2011$.

the final portion of the aorta. The aortic right arch does not emit any branch before its union with the left aortic arch, so the main visceral branches comes from the left aortic arch or directly from the aorta artery.

Keywords: Aorta arch, d'orbigny's slider turtle, morphology

\section{INTRODUÇÃO}

O tigre d'água brasileiro é um quelônio pertencente ao filo Chordata, subfilo Vertebrata, superclasse Tetrapoda, classe Reptila e ordem Chelonia (AMABIS; MARTHO, 1990; POUGH, HEISER, MCFARLAND, 1993; STORER et al., 1995). Representada por répteis conhecidos popularmente por tartarugas, cágados e jabutis, a ordem Chelonia é composta por duas subordens: Pleurodira e Cryptodira. A primeira é encontrada, atualmente, apenas no hemisfério sul, restrita ao habitat de água doce e constitui-se de três famílias (Chelidae, Pelomedusidae e Podocnemidae). Já a subordem Cryptodira possui o maior número de espécies viventes e habita todos os continentes com exceção da Antártida, sendo representada pelos jabutis, tartarugas límnicas e marinhas (POUGH et al., 1999).

Com a mais ampla distribuição dentre os quelônios da América, o gênero Trachemys se distribui desde Michigan, nos Estados Unidos, até a Argentina (SEIDEL, 2002). Na América do Sul, esse gênero apresenta duas disjunções: uma, com a espécie Trachemys adiutrix (VANZOLINI, 1995), a qual ocorre numa restrita e peculiar área do Maranhão e, a segunda, representada pela Trachemys dorbigni (DUMÉRIL; BIBRON, 1835) no extremo sul do continente, no estado brasileiro do Rio Grande do Sul e nos países Argentina e Uruguai (VANZOLINI, 1995; 1997).

Faria e Mariana (2001) descrevem que nos jabutis (Geochelone carbonaria), a origem dos arcos aórticos obedece ao padrão reptiliano, no 
KAMINISHI, A.P.S. et al. Comportamento da artéria aorta em Trachemys dorbigni (Duméril \& Bibron, 1835) (Testudines, Emydidae). PUBVET, Londrina, V. 5, N. 10, Ed. 157, Art. $1061,2011$.

qual a aorta direita deixa o coração pelo lado esquerdo e aorta esquerda pelo lado direito do ventrículo, posteriormente, cruzam-se e se voltam dorsalmente em sentido caudal. A aorta esquerda emite as artérias gástrica, celíaca e comunicante, sendo, que a artéria celíaca emite as artérias pancreaticoduodenal cranial, pancreaticoduodenal caudal, mesentérica caudal e mesentérica cranial.

Mediante a importância do conhecimento do sistema circulatório, em especial o comportamento da artéria aorta, que funciona como um meio de transporte do sangue rico em nutrientes, excretas, hormônios e componentes do sistema imunológico, objetivou-se descrever a origem e distribuição dos principais ramos da artéria aorta na cavidade celomática em Trachemys dorbigni.

\section{MATERIAL E MÉTODO}

Utilizaram-se dez exemplares de Trachemys dorbigni, cinco fêmeas e cinco machos, procedentes do acervo do Laboratório de Pesquisa em Animais Silvestres da Faculdade de Medicina Veterinária da Universidade Federal de Uberlândia.

Por dissecação na região cervical, a artéria aorta foi individualizada e canulada para realizar-se a lavagem do sistema vascular com solução fisiológica. Em seguida, injetou-se solução de neoprene látex "450" (Du Pont do Brasil S.A. - Indústrias Químicas) corada com pigmento específico (Globo S.A.-Tintas e Pigmentos). Para a fixação, utilizou-se solução de formol $10 \%$, injetada com seringas e agulhas por todo o corpo dos animais, posteriormente, os animais foram mantidos submersos em tanques contendo a mesma solução, por período mínimo de 96 horas. 
KAMINISHI, A.P.S. et al. Comportamento da artéria aorta em Trachemys dorbigni (Duméril \& Bibron, 1835) (Testudines, Emydidae). PUBVET, Londrina, V. 5, N. 10, Ed. 157, Art. $1061,2011$.

O plastrão foi retirado desprendendo-o da carapaça e das suas inserções na região cervical, nos membros e na cauda. Feito isto, as vísceras ficaram expostas e presas apenas na carapaça, que foi também aberta para que se pudesse observar a artéria aorta, bem como seus principais ramos. A partir deste momento procedeu-se a dissecação com pinças, bisturi e tesouras cirúrgicas e utilizou-se lupa Wild (10X) para melhor visualização das pequenas artérias. Focalizou-se a artéria aorta, a partir de sua origem até sua terminação registrando-se suas relações, e o comportamento de cada um dos seus ramos através de fotografias e esquemas.

\section{RESULTADOS}

Os resultados apresentados abaixo basearam-se em observações macroscópicas realizadas pela vista dorsal e ventral dos animais.

Analisando as peças dissecadas, pôde-se verificar que os arcos aórticos direito e esquerdo unem-se em um ponto, logo após o ventrículo cardíaco, obedecendo ao trajeto, inicialmente dorso-cranial e posteriormente ventrocaudal nas imediações da porção cardíaca do estômago. Próximo ao ponto de união dos dois arcos aórticos, dorsalmente a porção pilórica do estômago e cranialmente a este, o arco aórtico esquerdo emite ramos a irrigação desse órgão e para algumas vísceras da cavidade celomática, enquanto que o arco aórtico direito não emite nenhum ramo antes da sua união com o arco aórtico esquerdo. Após a união dos arcos aórticos ocorre a formação da artéria aorta, de onde se originam ramos para a região médio-caudal do corpo do animal.

Para melhor descrever os trajetos destes vasos, optou-se por fazê-lo separadamente desde sua origem no coração. 

\& Bibron, 1835) (Testudines, Emydidae). PUBVET, Londrina, V. 5, N. 10, Ed. 157, Art. $1061,2011$.

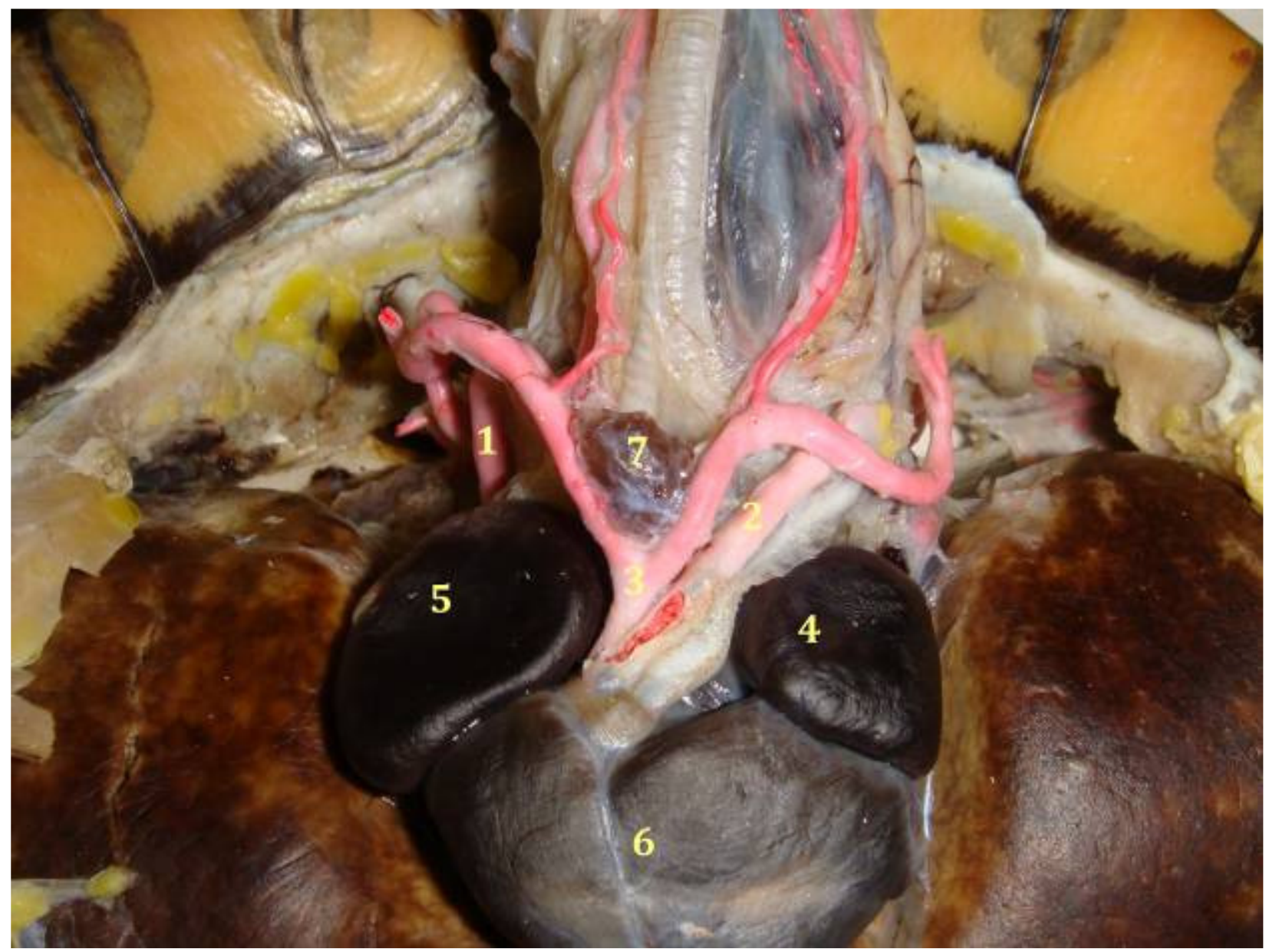

Figura 1: Vista ventral do coração de Trachemys dorbigni apresentando as principais estruturas visíveis.1- Arco aórtico direito, 2- Arco aórtico esquerdo, 3- Tronco Braquiocefalico, 4- Átrio esquerdo, 5- Átrio direito, 6- Ventrículo e 7- Tireóide.

\section{ARTÉRIA GÁSTRICA}

A artéria gástrica surge cranialmente ao ponto de união dos dois arcos aórticos, emergindo do arco aórtico esquerdo. Em 50\% dos casos, esse vaso emerge de um tronco comum formando as artérias gástrica e celíaca, sendo que em metade dos exemplares, eles surge cranialmente à origem da artéria celíaca e na outra metade, aparece na mesma altura da artéria celíaca e mesentérica cranial, em um tronco triplo. 
KAMINISHI, A.P.S. et al. Comportamento da artéria aorta em Trachemys dorbigni (Duméril \& Bibron, 1835) (Testudines, Emydidae). PUBVET, Londrina, V. 5, N. 10, Ed. 157, Art. $1061,2011$.

Após seu surgimento, a artéria gástrica emite um ramo esofágico, um ramo que se dirige para a região cardíaca do estômago e também a artéria gastroepiploica esquerda.

\section{ARTÉRIA CELÍACA}

A artéria celíaca possui pequeno calibre e origina-se a partir do arco aórtico esquerdo em sua porção terminal, passando ventralmente à artéria aorta, com a emissão de vários ramos a partir da região mediana. A artéria pancreaticoduodenal cranial é o primeiro ramo a emergir da face lateral da artéria celíaca, seguindo em direção ao pâncreas, em continuidade, seu trajeto ao estômago com a artéria gástrica direita e gastroepiplóica direita, com presença da fomração de anastomoses dessas estruturas com suas correspondentes do lado oposto em $100 \%$ dos casos.

\section{ARTÉRIA MESENTÉRICA CRANIAL}

Possui sua origem caudal à origem da artéria celíaca no arco aórtico esquerdo, anterior a união dos dois arcos aórticos, segue em direção ventrocaudal para irrigar o jejuno, ílio, ceco, colo ascendente e colo transverso. Há uma anastomose com a artéria mesentérica caudal originada da artéria ilíaca interna. 

\& Bibron, 1835) (Testudines, Emydidae). PUBVET, Londrina, V. 5, N. 10, Ed. 157, Art. $1061,2011$.

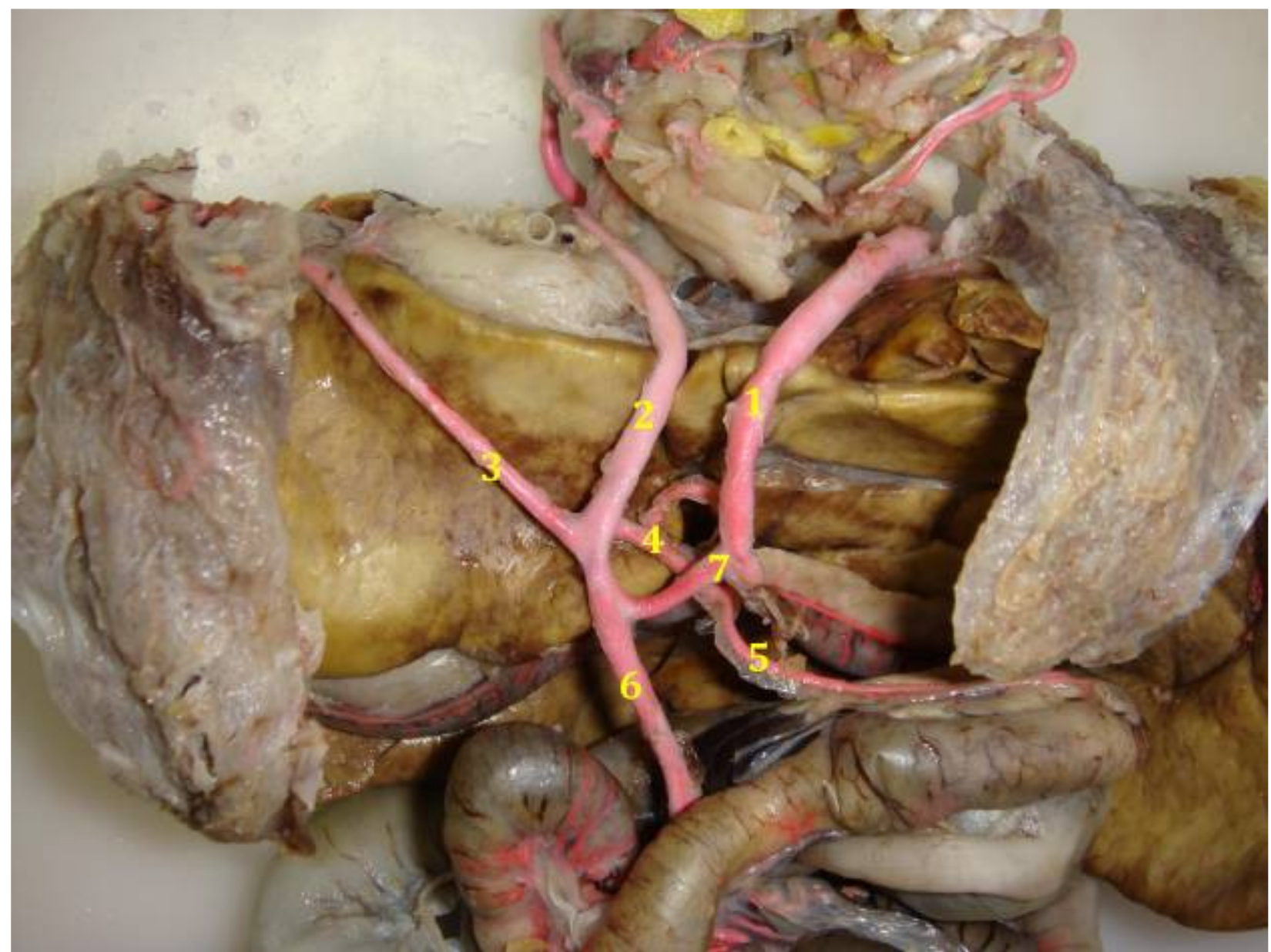

Figura 2: Vista dorsal da cavidade torácica de Trachemys dorbigni evidenciando os arcos aórticos e suas ramificações. 1- Arco aórtico direito, 2Arco aórtico esquerdo, 3- Artéria gástrica, 4- Artéria celíaca, 5- Artéria pancreaticoduodenal cranial, 6- Artéria mesentérica cranial e 7- União dos arcos aórticos formando a artéria aorta.

\section{ARTÉRIA AORTA}

Denomina-se de artéria aorta, a artéria que se inicia a partir da junção dos arcos aórticos esquerdo e direito. Representa um importante vaso no suprimento de sangue para a região médio-caudal do corpo do animal, uma vez que dela partem artérias para as vísceras, membros pelvinos, gordura lateral, músculos longitudinais, casco e cauda. 
KAMINISHI, A.P.S. et al. Comportamento da artéria aorta em Trachemys dorbigni (Duméril \& Bibron, 1835) (Testudines, Emydidae). PUBVET, Londrina, V. 5, N. 10, Ed. 157, Art. $1061,2011$.

\section{ARTÉRIAS RENAIS E GONADAIS}

Partindo da face ventro-lateral da artéria aorta, em direção aos rins, direito e esquerdo, observaram-se as artérias renais direita e esquerda variando de 3 a 6 ramos para cada rim. Em 50\% dos casos, surgiram ramos renais a partir da artéria ilíca comum esquerda e direita e nos demais casos esses ramos renais surgiam ventralmente à origem da artéria ilíaca comum esquerda e direita a partir da artéria aorta.

As artérias gonadais, chamadas ovarianas nas fêmeas ou testiculares nos machos, surgiram a partir das artérias renais, próximas ao hilo renal.

\section{ARTÉRIAS EPIGÁSTRICAS}

Acompanhando-se o trajeto da artéria aorta, logo após as artérias renais, encontra-se a origem das artérias epigástricas esquerda e direita. Estas seguiam em direção lateral esquerda e direita, respectivamente, e se bifurcavam em artérias marginocostal cranial e marginocostal caudal para seguir em direção cranio-lateral e caudo-lateral, respectivamente, irrigando as margens do casco e músculos pélvicos.

\section{ARTÉRIAS ILÍACAS EXTERNAS}

A artéria aorta, em sua porção terminal, emite dorso-lateralmente as artérias ilíacas externas esquerda e direita. As artérias ilíacas externas esquerda e direita dividem-se em artérias femoral e isquiática, irrigando os membros pelvinos.

\section{ARTÉRIAS ILÍACAS INTERNAS}

Surgem a partir da artéria aorta, ventro-lateralmente às artérias ilíacas externas esquerda e direita, destinando para as partes do sistema genital e bexiga urinária. A partir das artérias ilíacas internas, esquerda e direita, 
KAMINISHI, A.P.S. et al. Comportamento da artéria aorta em Trachemys dorbigni (Duméril \& Bibron, 1835) (Testudines, Emydidae). PUBVET, Londrina, V. 5, N. 10, Ed. 157, Art. $1061,2011$.

ocorre a origem da artéria mesentérica caudal, sendo que em $75 \%$ dos casos originou-se na artéria ilíaca interna direita, e em $25 \%$ dos casos da artéria ilíaca interna esquerda.

\section{ARTÉRIA CAUDAL}

A artéria caudal é um fino segmento que surge em $50 \%$ dos casos da extremidade caudal da artéria aorta, $25 \%$ dos casos da artéria ilíaca externa esquerda, proximal a sua origem na artéria aorta, e $25 \%$ dos casos a partir da artéria ilíaca externa direita, proximal à sua origem na artéria aorta.

\section{DISCUSSÃO}

O comportamento dos arcos aórticos nos exemplares de Trachemys dorbigni mostrou-se semelhante ao descrito por Neal e Rand (1954) que afirmam que estes arcos após deixarem o coração possuem um trajeto dorso-cranial e posteriormente ventro-caudal próximo à parte cranial do estômago, tendo um ponto de união entre os arcos aórticos direito e esquerdo, próximo a parte caudal do estômago.

Ashley (1955) e a presente pesquisa estão de acordo no que concerne à origem das artérias gástrica, celíaca e mesentérica cranial originárias do arco aórtico esquerdo. Faria e Mariana (2001), ao relatarem as artérias mesentéricas cranial e caudal como sendo originárias da artéria celíaca, contradizem os resultados encontrados em tigres d'água brasileiros, nos quais a artéria mesentérica cranial é proveniente do arco aórtico esquerdo e a artéria mesentérica caudal é proveniente da artéria ilíaca interna direita ou esquerda.

Neal e Rand (1954) e Faria (2000) relatam que o arco aórtico esquerdo origina a artéria celíaca. Ashley (1955) comenta que a mesentérica cranial 
KAMINISHI, A.P.S. et al. Comportamento da artéria aorta em Trachemys dorbigni (Duméril \& Bibron, 1835) (Testudines, Emydidae). PUBVET, Londrina, V. 5, N. 10, Ed. 157, Art. $1061,2011$.

também se origina do arco aórtico esquerdo, tal como a pesquisa aqui realizada, entretanto, Faria (2000) afirma que as artérias mesentéricas caudal e cranial apresentam-se como um segmento a partir da celíaca. No entanto, Faria (2000) utiliza a denominação artéria aorta esquerda ao invés de arco aórtico esquerdo e artérias mesentéricas inferior e superior, ao invés de artérias mesentéricas caudal e cranial.

Breland (1953) também afirma que a artéria celíaca origina-se do arco aórtico, sendo este denominado de arco sistêmico, não mencionando o arco aórtico esquerdo.

Contrariando os resultados encontrados em Trachemys dorbigni, Harrison (1964) descreve que é a partir da artéria aorta dorsal que se tem a artéria celíaca. $O$ autor comenta também que a artéria celíaca origina o ramo gástrico anterior, o ramo pancreaticoduodenal e o ramo hepático para os respectivos órgãos, e a artéria mesentérica anterior para o fígado e intestino delgado. Já a pesquisa presente demonstra ser o arco aórtico esquerdo o responsável pelo surgimento das artérias gástrica, celíaca e mesentérica cranial, sendo a artéria pancreaticoduodenal cranial originária da artéria celíaca.

Este trabalho descreve que a partir da união dos arcos aórticos direito e esquerdo tem-se a artéria aorta, entretanto, Baroudi (1965) relata a presença de duas aortas antes da união das mesmas, e após esta união encontra-se a artéria torácica única que emite troncos e irriga as estruturas do organismo. Storer et al. (1995) comentam a existência de dois arcos aórticos que se unem para formar a aorta dorsal, assemelhando-se às observações em Trachemys dorbigni, que apontam para a presença de uma artéria aorta.

O presente trabalho concorda com Rodrigues et al. (2003), quanto a origem das artérias gástrica, celíaca e mesentérica cranial serem no arco 
KAMINISHI, A.P.S. et al. Comportamento da artéria aorta em Trachemys dorbigni (Duméril \& Bibron, 1835) (Testudines, Emydidae). PUBVET, Londrina, V. 5, N. 10, Ed. 157, Art. $1061,2011$.

aórtico esquerdo e a artéria mesentérica caudal ser proveniente da artéria ilíaca interna direita ou esquerda.

Santos et al. (2004) relatam em estudo com Podocnemis expansa que a artéria mesentérica cranial é um ramo do tronco celíaco-mesentérico e origina as artérias pancreaticoduodenal caudal, ileocólica e jejunais, o que não foi comprovado em nosso experimento, pois não foi constatada a presença de um tronco celíaco-mesentérico e a artéria mesentérica cranial tendo origem diretamente do arco aórtico esquerdo.

Referente à origem das artérias gonadais em jabuti, Faria e Mariana (2001), concordam com os achados nesse experimento, quando dizem que as artérias testiculares ou ovarianas surgiram, em $100 \%$ dos casos, a partir das artérias renais, próximo ao hilo renal. Hirano et al. (2009) encontraram dois pares de ramos renais partindo em sua maioria da face dorsal da artéria aorta, o que condiz em parte com o experimento feito com $T$. dorbigni, no qual foram observados de três a seis ramos para cada rim.

\section{CONCLUSÕES}

Com base nos dados do presente estudo com Trachemys dorbigni, conclui-se que:

1- A origem dos arcos aórticos obedece ao padrão reptiliano, onde o arco aórtico direito deixa o coração pelo lado esquerdo e o arco aórtico esquerdo pelo lado direito do ventrículo.

2- O arco aórtico esquerdo origina as artérias gástrica, celíaca e mesentérica cranial.

3- A artéria celíaca emite a artéria pancreaticoduodenal cranial, artéria gástrica direita e artéria gastroepiploica direita. 
KAMINISHI, A.P.S. et al. Comportamento da artéria aorta em Trachemys dorbigni (Duméril \& Bibron, 1835) (Testudines, Emydidae). PUBVET, Londrina, V. 5, N. 10, Ed. 157, Art. 1061, 2011.

4- A artéria aorta emite as artérias renais, epigástricas, ilíacas externas, ilíacas internas e em $50 \%$ dos casos a artéria caudal.

\section{REFERÊNCIAS BIBLIOGRÁFICAS:}

ASHLEY, L. M. Laboratory Anatomy of the Turtle. Dubuque: WM. C. Brown, p. 26-34, 1955. BAROUDI, R. Elementos de Zoologia de Cyclostomata a Mammalia. 4. ed. São Paulo: Nobel, p. 67, 1965.

BRELAND, O. P. Manual of Comparative Anatomy. 2. ed. Nova York: McGraw-Hill Book, p. $124,1953$.

FARIA, T. N. Descrição da origem, trajeto e número das principais artérias do jabuti "Geochelone carbonaria"(Spix, 1824). 2000. 42 p. Dissertação (Mestrado em Anatomia dos Animais Doméstico). Universidade de São Paulo, São Paulo. 2000.

FARIA, T. N; MARIANA, A. N. B. Origens e ramificações das artérias aortas esquerda e dorsal do jabuti (Geochelone carbonaria,Spix, 1824). Brazilian Journal of Veterinary Research and Animal Science, v. 38, n. 4, p. 155-159, 2001.

HARRISON, B. M. Manual of Comparative Anatomy a General Laboratory guide. 2. ed. St. Louis: C. V. Mosby, p. 156, 1964.

HIRANO, L. Q. L.; SANTOS, A. L. Q.; PEREIRA, P. C.; SilVA, J. M. M.; KAMINISHI, A. P. S.; FERREIRA, C. G. Anatomia Vascular das Artérias Renais e Gonadais de Podocnemis unifilis. Acta Scientiarum Biological Sciences, v. 31, n. 2, p. 191-194, 2009.

NEAL, H. V.; RAND, H. W. Comparative Anatomy. New York: Blakiston Company, p. 356, 1954.

POUGH, F. H.; HEISER, J. B.; MCFARLAND, W. N. A Vida dos Vertebrados. São Paulo: Atheneu, p. 386- 409, 1993.

POUGH, F. H.; HEISER, J. B.; McFARLAND, W. N. A vida dos vertebrados., São Paulo: Atheneu Editora, 1999. 798p.

SANTOS, A. L. Q.; MUNDIM, A. V.; MORAES, F. M.; ALVARENGA, G. J. R.; MARQUES, F. K.; BORGES, K. M.; ALVES JUNIOR, J. R. F. Origem e Ramificações das Artérias Mesentéricas Cranial e Caudal em Tartaruga da Amazônia Podocnemis expansa. Archives of Veterinary Science, v. 9, n. 2, p. 49-53, 2004.

SEIDEL, M. E. Taxonomic observations on Extant species and subspecies of Slider Turtles, genus Trachemys. Journal of Herpetology, v. 36, n. 2, p. 285-292, 2002.

STORER, T. I.; USINGER, R. L., STEBBINS, R. C.; NYBAKKEN, J. W. Zoologia Geral. São Paulo: Nacional, p. 267-289, 1995.

VANZOLINI, P. E. A new species of turtle, genus Trachemys, from the state of Maranhão, Brazil (Testudines, Emydidae). Revista Brasileira de Biologia, v. 55, n. 1, p. 111-125, 1995.

VANZOLINI, P. E. A note on the reprodution of Trachemys dorbigni (Testudines, Emydidae). Revista Brasileira de Biologia, v. 57, n. 2, p. 165-175, 1997. 